\title{
UDIO DE LA NORMATIVIDAD DE LOS ESPACIOS INFORMÁTICOS ACADÉMICOS EN IBEROAMÉRICA
}

\section{STUDY OF THE REGULATION OF ACADEMIC COMPUTING SPACES: PANORAMA OF SPAIN, PORTUGAL, AND LATIN AMERICA}

\author{
Javier Fombona Cadavieco; fombona@uniovi.es \\ Universidad de Oviedo \\ MariCarmen González Videgaray; , mcgv@unam.mx \\ Universidad Nacional Autónoma de México \\ María de Fátima Goulao; fatimapgoulao@gmail.com \\ Universidade Aberta de Portugal
}

\section{RESUMEN}

Las instituciones educativas ofrecen aulas informáticas reguladas por normas. Su estudio ilustra sobre las características tecnológicas, las intenciones didácticas y el comportamiento de sus usuarios. Este trabajo analiza en profundidad un centenar de reglamentos en instituciones de diversos niveles educativos en países Iberoamericanos. Las normas se categorizaron y contrastaron, identificando aspectos prioritarios consensuados tales como la obligación de identificar al usuario, las prohibiciones de instalar software, comer en estos espacios o jugar en los equipos.

PALABRAS CLAVE: Aplicaciones informáticas, aulas virtuales, comportamiento, normativa informática.

\section{ABSTRACT}

Most educational institutions offer computer labs regulated by norms. Their study can shed light on didactic intentions, user behavior and technological characteristics of the computer lab. In this paper we study a hundred regulations obtained from Internet, used by institutions of different countries and levels. The rules were categorized and contrasted, identifying priority issues as "user identification required", "prohibition of software installing, of eating at this place, and playing on computers".

KEYWORDS: Computer software, virtual classrooms, behavior, ICT Policy. 


\section{INTRODUCCIÓN}

Las instituciones educativas suelen tener una o varias aulas dedicadas a los recursos informáticos, llamados salones o laboratorios de cómputo en lberoamérica, cuyas funciones cambian con el tiempo (Farah, 2005). Inicialmente, se instalaban equipos para estudiar lenguajes de programación. Luego se introdujeron ordenadores o computadoras personales y se amplió su uso con la aparición utilidades amigables, procesadores de textos, hojas de cálculo y gestores de presentaciones de diapositivas. Este incremento de posibilidades también apoyó el aprendizaje en materias diversas. Hoy, el número elevado de aplicaciones y la conjunción con Internet han convertido estas herramientas en medios de comunicación interactivos y dinámicos (Acikalin, 2010). Se posibilita observar videos educativos en la red o consultar bases de datos de revistas especializadas en sitios como Eigen Factor, DOAJ, SClelo o Redalyc, pero sobre todo se crean nuevas metodologías y entornos de aprendizaje (López, et al., 2012; González-Videgaray, et al., 2009).

Por otra parte, la tecnología ha disminuido su costo, y se vuelve más potente y portátil. La confluencia entre diversidad de equipos y funciones han hecho que la figura original de los laboratorios de cómputo se desdibuje y se conforme de maneras diferentes.

Las instituciones educativas son las responsables de que los espacios educativos genéricos y los específicamente informáticos cumplan sus funciones y se haga uso óptimo de los recursos allí dispuestos. Por ello es de interés observar cómo funcionan estos espacios y cómo promueven el aprendizaje de los estudiantes. Sin embargo, existe poca literatura al respecto, esta carencia se ha detectado en el contexto latinamericano según la indagación del equipo de investigación de la UNAM, Portugal (Universidade Aberta) y España (Universidad de Oviedo).

Se ha hecho un acercamiento a la situación de los laboratorios de informática a través del estudio de sus reglamentos, ya que en ellos se reflejan las conductas y preocupaciones predominantes, así como las modificaciones que ha sufrido la tecnología utilizada. Con este estudio se pretende observar qué ocurre en la práctica dentro de estos laboratorios educativos y qué acciones pueden considerarse adecuadas para facilitar la gestión y el cumplimiento de los objetivos en este tipo de espacios.

\subsection{Estado de la cuestión}

Esta investigación se orientó a describir las normativas existentes en espacios educativos informáticas en Iberoamérica. En la revisión de la literatura destacan los trabajos que analizan el uso de los recursos informáticos en los niveles educativos iniciales (Pedrosa, 2001), en educación secundaria (Iscioglu, 2011), post-secundaria (Lowerison, et al., 2006), en niveles superiores (Selwyn, 2007) o con la perspectiva de género (Anguita y Ordax, 2000). También hay experiencias que describen la relación entre el docente y estos recursos en su integración curricular (Biscomb, Devonport y Lane, 2008; Martin, Picos y Egido, 2010).

Existen análisis sobre la privacidad de los contenidos (Kerr, 2011) y sobre la normativa de seguridad en Internet (Flowers y Rakes, 2000; Rolando y Salvador, 2009; Sureda, Comas, et al. 2010, Gutiérrez, et al., 2010; Sureda Negre, et al., 2010). Otros trabajos analizan el diseño de estos espacios comunes (Gibert, 2006; Oyewole, 2010). Pero prácticamente no se han encontrado estudios sobre las normativas de los espacios informáticos educativos. 


\subsection{Los reglamentos educativos e informáticos}

La finalidad de un reglamento es articular normas que controlen la actividad y el comportamiento de las personas en una institución, para poder crear un ambiente de convivencia y lograr unos objetivos. Un reglamento se compone de proposiciones que señalan una conducta a seguir o evitar, también incluye sanciones ante incumplimientos de las anteriores.

La aplicación de las reglas puede debe respetar las normativas o legislaciones superiores. Y estos referentes deben ser considerados por los encargados tanto de establecer como de aplicar las normas.

Los centros educativos integran personas con perfiles y orígenes sociales y culturales diversos, esta circunstancia potencia los reglamentos unificadores de conductas. Por ello, las normas suelen ser públicas y se plasman en los documentos de régimen interno de cada centro escolar. Se dan a conocer en forma impresa, electrónica o ambas.

En estos espacios muchos integrantes de la comunidad académica deben compartir el mismo recurso informático, cuyas características lo hacen susceptible de usos no apropiados, averías y distorsiones de los objetivos educativos marcados.

Asimismo, las instituciones cuentan con recursos limitados lo que implica un cuidado de estas salas de informática, su hardware, software y mobiliario. En esta línea, se ha de considerar que la actividad docente tiene como meta la formación de las personas. Esto imprime un carácter específico a sus reglamentos, que deben ser flexibles, adaptables y sujetos al razonamiento sobre los valores que van asociados con cada norma, tales como el respeto a los demás, el uso cuidadoso de los recursos y el bien común, entre otros. Por ello debería privilegiar la función correctiva y formadora sobre la punitiva.

Los reglamentos pueden ser precisos o abiertos a la libre interpretación; cortos o extensos; específicos o genéricos. Parece que se obtienen buenos resultados cuando las normas responden a un diseño eficaz (Garland y Noyes, 2004). En todo caso son un intento de dar pautas para las conductas y tareas encaminadas a lograr objetivos relacionados con el desarrollo de la persona.

En otras ocasiones estas normas se dan por conocidas y aunque no estén escritas se cumplen de forma voluntaria (Schraw, 2010).

El reglamento, concreta un conjunto de pautas para corregir un problema existente o prever un problema eventual así como una posible desviación de los objetivos planteados. Sin embargo, los modelos normativos se enriquecen con eventos que no habían sido previstos y que van ocurriendo en la vida comunitaria (Koh y Frick, 2009). De ahí que muchos reglamentos crezcan y se reorientan con el tiempo, reflejando los comportamientos habituales de los usuarios.

\subsection{Contexto dinámico de los espacios informáticos}

El objetivo inicial de la creación de las aulas o laboratorios de cómputo fue ofrecer a los estudiantes los equipos y el software requeridos por sus asignaturas. Las salas informáticas educativas se concebían aisladas, pero luego se conectaron a redes locales para compartir 
impresoras $u$ otros servicios. Posteriormente se abrieron a las comunicaciones externas para tener acceso a Internet o a redes regionales, Intranets. Esta ecología determina un lugar de trabajo dinámico (Gutiérrez, et al., 2010). Hoy se incorporan computadoras personales independientes y conexiones inalámbricas a la red que desdibujan la frontera específica del aula de informática (Young, 2008). Estos aspectos deben considerarse para una gestión adaptativa de los espacios informáticos: mobiliario versátil, conexiones eléctricas, accesos WIFI y la opción de acceder a otros recursos a través de un servidor potente.

El espacio educativo se ha transformado aún más y hoy se incorporan ambientes virtuales (Locatis, et al., 2008) donde estudiantes y profesores no coinciden en un lugar, sino que se conectan a Internet y se comunican de forma asincrónica o en tiempo real, sincrónico.

El software es accesible y variado, presentándose alternativas de software libre (Elliott y Scacchi, 2005) y sitios web de acceso abierto (Lund University Libraries, 2009) que multiplican las opciones para trabajar, manipular estas aplicaciones e interactuar con nuevos planteamientos educativos.

Aunque los contextos son difíciles de acotar, los centros escolares necesitan ofrecer a sus alumnos la posibilidad de usar determinado hardware y software con propósitos específicos. Por ello es conveniente utilizar computadoras propias de la institución, conectadas o no a un servidor, con cierto diseño y configuración de un laboratorio de cómputo, con equipos a disposición del alumnado, y con una orientación didáctica. En este trabajo nos referiremos a las normas de uso para este tipo de espacios.

\subsection{Objetivos}

Observamos una laguna en el conocimiento sobre la forma de gestión de los espacios educativos de cómputo en Iberoamérica. Consideramos que esta actividad se resume de manera objetiva en los reglamentos y normativas usados para el funcionamiento de los laboratorios informáticos. Estos documentos abordan los principales problemas en cada caso y reflejan el comportamiento habitual de los usuarios. Los reglamentos y normativas también muestran la intencionalidad de quienes los administran, así como las características tecnológicas predominantes.

Esta investigación tiene por objetivo mostrar en el contexto iberoamericano el funcionamiento de los espacios educativos comunes con recursos informáticos a través del estudio descriptivo de sus normas. Para analizar los protocolos de funcionamiento eficaz hemos recopilado las normas contrastadas y experimentadas previamente. Se pretende mostrar la relación entre los equipos informáticos y sus usuarios, sus usos predominantes, las conductas deseables y las preocupaciones de los responsables de la gestión docente.

A partir de este estudio se podría mejorar la toma de decisiones en este ámbito, plantear nuevos reglamentos o investigaciones que, más adelante, evalúen las modificaciones de estas normas con respecto al tiempo, a los cambios tecnológicos y a los nuevos hábitos de los usuarios. 


\section{METODOLOGÍA}

\subsection{Muestra}

Se recopilaron normas que regulasen cualquier espacio con recursos informáticos dispuestos para ser utilizados principalmente con fines educativos. Debían de estar escritas en español o portugués, bajo el supuesto de que son contextos idiomáticos afines. Y tendrían que estar disponibles en Internet en 2011.

Se consideraron 362 páginas web, y de su análisis en profundidad se seleccionaron 100 como significativas, válidas y fiables que se corresponden con los países mostrados en la Tabla 1. El resto se desecharon por: no estar relacionadas con el ámbito educativo (79), tener referencias incompletas (29), no ser un reglamento (55), mostrar incoherencia en la redacción (23), repetir contenidos (46) o provenir de un sitio web no estable (10).

\begin{tabular}{lc}
\hline País & № de sitios web \\
\hline Argentina & 1 \\
Brasil & 4 \\
Chile & 3 \\
Colombia & 11 \\
España & 71 \\
México & 1 \\
Paraguay & 1 \\
Portugal & 6 \\
Uruguay & 2 \\
Total & 100 \\
\hline
\end{tabular}

Tabla 1. Países originarios de los reglamentos.

Se diferenció el espacio educativo referido a: Educación Infantil y Primaria, Enseñanza Secundaria, Bachiller, Formación Profesional, nivel Universitario y otros ámbitos (Tabla 2), pudiendo incidir un reglamento en varios espacios educativos.

\begin{tabular}{lr}
\hline Espacio educativo & Frecuencias \\
\hline Educación Infantil y Primaria & 27 \\
Enseñanza Secundaria & 31 \\
Bachiller & 16 \\
Formación Profesional & 9 \\
Universidad & 46 \\
Ámbito no reglado (bibliotecas, centros municipales, etc.) & 9 \\
\hline
\end{tabular}

Tabla 2. Ámbitos a los que hacen referencias las normas.

\subsection{Instrumento de investigación}

El instrumento de investigación utilizado consistió en un cuestionario dividido en dos secciones. La inicial, ya señalada, caracteriza el nivel educativo y la tipología del espacio al que afectan las normas. La segunda, delimita las categorías específicas de cuantificación de las normas:

- Forma de acceso a los recursos informáticos.

- Normas de impresión.

- Obligaciones de los usuarios. 
- Prohibiciones sobre: Uso del software; Uso del hardware y equipos informáticos; Acceso a la red e Internet; Convivencia en este espacio.

- Procedimientos ante incidencias.

- Penalizaciones.

Cada ítem engloba un conjunto de respuestas que no son mutuamente exclusivas. Para abrir la posibilidad a incluir nuevas normas se colocó la opción "Otra" al final de cada cuestión. Asimismo se contabilizó el número de normas por cada reglamento localizado.

Estos ítems han sido validados fruto del estudio de un conjunto representativo de documentos relativos a esta temática, analizados separadamente por cada miembro del equipo investigador y fueron seleccionados aquellos ítems que reunían un mayor consenso.

\subsection{Procedimientos}

Durante 2011 se seleccionaron los sitios web y su respectiva catalogación. El instrumento de análisis fue construido en formato electrónico para permitir una rápida recogida de datos.

Esta estructura de recogida de datos fue sometida a un pre-test para aumentar la validez, y antes de configurar una versión definitiva. Así, fue solicitada la valoración a un conjunto de académicos cualificados sobre los siguientes dos aspectos:

- Pertinencia / adecuación de las opciones y

- Correcta comprensión de los enunciados.

Tras esta evaluación se procedió a reestructurar el cuestionario de acuerdo con las sugerencias aportadas, hasta llegar al instrumento definitivo.

Los sitios web fueron analizados de acuerdo con este diseño y los datos recogidos dan lugar a los resultados que se presentan en el siguiente apartado.

\section{RESULTADOS}

El promedio de normas en los reglamentos es cercano a 15, con poca variación entre los niveles educativos (Tabla 3), con excepción del incremento en la formación profesional de grado medio.

\begin{tabular}{lc}
\hline Nivel educativo & № promedio de normas \\
\hline Educación primaria & 10.72 \\
Enseñanza secundaria & 15.81 \\
Bachiller & 14.92 \\
Formación profesional grado medio & 29.00 \\
Formación profesional grado superior & 16.25 \\
Universidad & 15.85 \\
Otros (centro municipal, biblioteca, etc.) & 12.14 \\
Total & 15.01 \\
\hline
\end{tabular}

Tabla 3: Promedio de normas por espacio.

Se cuantificaron las opciones posibles de acceso a los equipos y documentos (Tabla 4) y se registraron las frecuencias que se habían elegido para cada caso con un máximo de 100 . La 
norma con mayor número de ocurrencias es "la solicitud de identificación para tener acceso al laboratorio".

\begin{tabular}{lr}
\hline Norma & Frecuencia \\
\hline Acceso con credenciales y documentos identificativos. & 58 \\
Obligación de guardar los documentos en dispositivos externos. & 35 \\
Obligación de apagar el equipo al final (no se apaga el monitor). & 35 \\
Hay establecido un número limitado máximo de usuarios por equipo. & 30 \\
Posibilidad de guardar documentos en el equipo o servidor. & 23 \\
Obligación de no apagar equipo, cerrar sesión, cerrar programas (salvo última sesión en & 22 \\
que se apagarán de forma correcta: inicio-apagar sistema-aceptar). & \\
Hay un equipo/ordenador específico para cada alumno. & 14 \\
Uso de equipos autónomos sin servidor. & 12 \\
Hay un registro y monitorización de toda la actividad informática del usuario. & 5 \\
Hay una edad mínima de acceso al aula. & 1 \\
\hline
\end{tabular}

Tabla 4: Protocolos de acceso a equipos informáticos y a los documentos.

\begin{tabular}{lr}
\hline Norma & Frecuencia \\
\hline Sin referencias ni normas de impresión. & 69 \\
Impresión libre si el alumnado aporta papel o tinta. & 7 \\
Se cobra un dinero por cada impresión. & 7 \\
Hay un número de impresiones gratuito. & 6 \\
Uso de impresoras autorizado por el profesor. & 6 \\
Impresión prohibida. & 5 \\
Impresión libre. & 4 \\
Prohibido bloquear las colas de impresión. & 3 \\
\hline
\end{tabular}

Tabla 5: Protocolos de Impresión.

En cuanto a las normas de impresión, puede verse (Tabla 5) que en la generalidad de los casos, el 69\%, no hay normas para este rubro.

Entre las obligaciones generales del usuario destaca en el $42 \%$ la reserva previa de los equipos por parte del profesor (Tabla 6). Casi en seguida está la obligación de identificarse si así lo requiere el responsable del aula informática.

\begin{tabular}{lr}
\hline Norma & Frecuencia \\
\hline Obligación de reserva previa de los equipos, ante el profesor. & 42 \\
Obligación de identificarse si lo requiere el profesor/responsable. & 40 \\
Obligación de reserva previa del equipo, por el alumno. & 36 \\
Prohibido el acceso de personas ajenas al centro. & 34 \\
Limitación de tiempo máximo de uso diario. & 31 \\
Obligación de conocer las normas, funcionamiento del ordenador y programas a & 6 \\
utilizar. & \\
Obligación de registro inicial en un documento escrito. & 5 \\
Obligación de comprobar el estado inicial del aula. & 5 \\
Prohibido usar aula de ordenadores durante las guardias. & 4 \\
Obligación de buen uso, cada alumno es responsable del mantenimiento de su equipo & 3 \\
informático. & \\
\hline
\end{tabular}

Tabla 6: Obligaciones generales del usuario. 
La Tabla 7 contiene las normas relativas al software, dentro del subgrupo de prohibiciones. Aquí destaca la norma con mayor porcentaje de ocurrencia en todos los reglamentos revisados: la prohibición de instalar software o programas sin autorización, con un $88 \%$ de ocurrencias. Otra norma con alto porcentaje de registros es la relativa a la prohibición de modificar el software de red instalado o los accesos y sus contraseñas.

\begin{tabular}{lr}
\hline Norma & Frecuencia \\
\hline Prohibido instalar software o programas sin autorización. & 88 \\
Prohibido modificar el software de red o accesos y sus contraseñas. & 62 \\
Prohibido que los alumnos compartan credenciales o accesos como usuarios & 28 \\
distintos al verdadero. & \\
Prohibido modificar configuración, fondos de pantalla o inicio de Internet. & 26 \\
Prohibido eliminar documentos de otros usuarios. & 23 \\
Prohibido guardar documentos ilegales u ofensivos. & 4 \\
Prohibido guardar cualquier documento. & 2 \\
Prohibida cualquier cosa que no sea didáctica. & 2 \\
\hline
\end{tabular}

Tabla 7: Normas sobre el uso del software.

Dentro de la Tabla 8 se organizan las normas relacionadas con el hardware y los equipos, los instrumentos informáticos y el cableado.

\begin{tabular}{lr}
\hline Norma & Frecuencia \\
\hline Prohibido tocar los interruptores o los cables. & 14 \\
Prohibido manipular el hardware del equipo y el resto sin cuidado (CPU, monitores, & 10 \\
etc.). & \\
Prohibido conectar dispositivos de memoria externa USB o discos, CD o DVD externos. & 6 \\
Prohibido desconectar los equipos. & 4 \\
\hline
\end{tabular}

Tabla 8: Normas sobre el uso del hardware.

La Tabla 9 agrupa normas sobre el uso de Internet. Destaca la prohibición de jugar en línea, usar los chat y explorar páginas no didácticas.

\begin{tabular}{lr}
\hline Norma & Frecuencia \\
\hline Prohibido usar programas de juegos en línea. & 45 \\
Prohibido usar programas de chat. & 45 \\
Prohibido explorar páginas web no didácticas. & 43 \\
Prohibido hacer descargas ilegales de material protegido por autor, tipo Emule o Peer & 40 \\
to Peer. & \\
Prohibido usar la red para molestar a otros usuarios. & 32 \\
Prohibido usar redes sociales: Facebook, Twitter, etcétera, salvo autorización expresa. & 32 \\
Prohibido cualquier acoso, calumnia o difamación. & 30 \\
Sin normativa sobre Internet. & 14 \\
Prohibido el acceso a páginas web con contenido sexual o erótico. & 11 \\
Prohibido crear cuentas de usuario o de correo electrónico. & 10 \\
Prohibido comerciar en Internet. & 9 \\
Prohibido conectarse a Internet sin autorización y/o control del profesor responsable. & 6 \\
Prohibido acceder a páginas de contenido político, racista o religioso. & 6 \\
Prohibido el acceso a páginas web con contenido violento. & 4 \\
Prohibido descargar documentos “pesados” (música, películas...). & 4 \\
Prohibido virus, troyanos, etcétera. & 4 \\
Prohibido acceder a contenidos ilícitos en la web. & 3 \\
\hline
\end{tabular}




\begin{tabular}{lr}
\hline Norma & Frecuencia \\
\hline Permitida la descarga con supervisión del tutor. & 2 \\
Prohibida mensajería a móviles. & 1 \\
Prohibido hacer copias ilegales. & 1 \\
Prohibido mandar fotografías. & 1 \\
\hline
\end{tabular}

Tabla 9: Normas relativas al uso de Internet.

En las normas relativas a la convivencia (Tabla 10) destaca la prohibición de comer o beber, que aparece en el $72 \%$ de los reglamentos, y la prohibición de usar juegos aparece en el $45 \%$.

\begin{tabular}{lr}
\hline Norma & Frecuencia \\
\hline Prohibido comer o beber. & 72 \\
Prohibido usar juegos. & 45 \\
Prohibido mover los equipos, mesas, etcétera. & 43 \\
Obligación de limpieza, colocar las pertenencias sillas y equipos de forma ordenada, & 35 \\
especialmente al finalizar cada sesión. & \\
Prohibido hablar y hacerlo de forma molesta. & 33 \\
Prohibido sacar los equipos del aula sin autorización. & 24 \\
Prohibido reproducir sonido y música en volumen alto (no individualizado). & 24 \\
Prohibido estar en ese espacio en el periodo de clases. & 16 \\
Prohibida la telefonía celular. & 15 \\
Prohibido estar en el aula de informática sin la presencia del profesor/coordinador del & 4 \\
aula. & \\
Prohibidos comportamientos ofensivos o agresivos. & 3 \\
Prohibido escuchar música. & 2 \\
Prohibido entrar con objetos personales (mochila, portafolios, etcétera.) & 2 \\
Prohibidos los animales, salvo que acompañen a invidentes. & 2 \\
Prohibido usar la sala como lugar de estudio u otras tareas personales. & 1 \\
\hline
\end{tabular}
Tabla 10: Normas relativas a la convivencia.

En los procedimientos a seguir ante averías o incidencias (Tabla 11) la mayoría de los reglamentos (59\%) optan por el aviso verbal.

\begin{tabular}{lr}
\hline Norma & Frecuencia \\
\hline - Aviso verbal al coordinador o profesor. & 59 \\
- No hay descrito procedimiento ante incidencias. & 21 \\
- Cubrir datos en una hoja de incidencias y entregarla al coordinador o profesor. & 15 \\
- Obligación avisar la presencia de anomalías. & 10 \\
- Ante una avería consecuencia de un mal uso, deberá de hacerse cargo & 10 \\
económicamente de su reparación. Estará implicado el último usuario o en caso de que & \\
se desconozca el causante de los daños, la clase entera deberá de hacerse cargo. & \\
- Obligación del profesor de controlar el estado del aula y avisar al coordinador si hay & 5 \\
incidencias. & \\
- Cubrir datos en una hoja de incidencias y enviarla por email al coordinador o profesor. & 2 \\
- Rellenar un formulario de incidencias en página web. & 2 \\
\hline
\end{tabular}

Tabla 11: Procedimientos a seguir en caso de averías o incidencias.

En los registros sobre las penalizaciones (Tabla 12) destacada la suspensión temporal (37\%) y definitiva (30\%) del acceso al aula. En una tercera parte de los reglamentos no se describen sanciones. 


\begin{tabular}{lr}
\hline Norma & Frecuencia \\
\hline Suspensión temporal de acceso al aula. & 37 \\
No hay descritas sanciones. & 33 \\
Suspensión definitiva de acceso al aula. & 30 \\
Aplicación del reglamento disciplinario de la institución. & 29 \\
Reposición del material dañado o averiado. & 20 \\
Amonestación verbal. & 13 \\
Suspensión de la conexión a Internet. & 4 \\
Amonestación escrita. & 4 \\
Sanción económica. & 3 \\
Sanción indefinida. & 1 \\
\hline
\end{tabular}

Tabla 12: Penalizaciones.

\section{DISCUSIÓN}

Se observa que los reglamentos son breves y están formados por proposiciones claras y específicas. La mayoría de las normas están relacionadas con Internet y la preocupación más destacada se refiere a prohibir la instalación de software sin autorización.

Los recursos informáticos aparecen vinculados a un equipo servidor central o se encuentran independientes, estas opciones ser relacionan con la posibilidad de guardar los documentos en ese servidor, en el equipo individual o en dispositivos externos físicos o virtuales (on-line). La autentificación del usuario y su perfil singular ante un servidor conlleva una responsabilidad específica de la gestión de su software y sus documentos de trabajo. Se puede observar cómo la utilización de un servidor centralizado posibilita realizar tareas colaborativas (Anguita y Ordax, 2000; Inan, et al. 2010) y compartir recursos comunes tales como impresoras. Algunos inconvenientes sobre el uso de equipos centralizados en red indican limitaciones en su velocidad y capacidad de almacenamiento (Selwyn, 2009), por ello en ocasiones se decide que el alumnado tenga los accesos directos sin precisar una contraseña de inicio.

Las normas relativas a la impresión de documentos señalan que se utiliza una impresora en red para dar servicio a varios equipos. Sorprende que no existan muchas reglas lo cual sugiere que los usuarios manejan más archivos digitales que impresos en papel, o también que los usuarios imprimen los documentos en sus hogares o en otro sitio.

Las obligaciones generales del usuario, como identificarse (40\%), evitar el acceso de personas ajenas al centro (34\%), limitar el tiempo de uso (31\%), reserva de los equipos por parte del profesor (42\%) y el alumno (36\%), están relacionadas con el hecho de que se trata de recursos relativamente caros y limitados (Bozionelos, 2004). Estas acciones ayudan también a mantener un registro de las actividades, momentos de mayor demanda, nivel de funcionamiento singular de equipos, personas que los utilizan, etcétera.

Existe una marcada preocupación sobre la manipulación del software, ya que esto puede implicar trastornos en la configuración de los equipos, instalación de programas inadecuados o virus dañinos. Sobre todo, se observa esta limitación en el nivel educativo inicial, mientras que en el superior puede ser interesante que el alumnado gestione con mayor libertad estos recursos (Tondeur, van Braak y Valcke 2007). Ciertos autores han sugerido la existencia de 
una generación de nativos digitales (Bennett, et al., 2008; Prensky, 2001; Selwyn, 2009) o net generation (Carlson, 2005; Judd y Kennedy, 2011), y argumentan a favor de un alumno con capacidad de gestión informática plena (Bowman, et al., 2010; Willingham, 2010; Junco y Cotten, 2011).

Las prohibiciones relacionadas con Internet se centraron en el uso de programas de juegos (45\%), exploración de webs no didácticas (43\%), uso programas de chat (45\%) y descarga de material protegido por derechos de autor (40\%). El equipo conectado a una red y a Internet aumenta su potencial como medio de investigación, gestión de datos y adquisición de determinadas competencias (Flowers y Rakes, 2000; Witten, et al., 2009), pero también supone la entrada a un contexto en el que abundan otros objetivos no educativos, desde lúdicos o comerciales, hasta ilícitos, pornográficos y agresivos. Una solución es la navegación controlada a través de la exploración previa de páginas a consultar (p.e. webquest) o la monitorización en tiempo real de la actividad del alumnado, aunque esto puede traer consigo problemas de ética y privacidad (Cox, et al., 2005).

Se detectaron también normas de convivencia comunes al resto de actividad docente (Shell y Husman, 2008) como: prohibido comer, beber (72\%) o usar juegos (45\%), mover los equipos y mobiliario (43\%); obligación de orden y limpieza (35\%).

Se observan reglas relativas al control de la agresividad dentro del aula informática o cyberbullying (Li, 2007; Kowalski, et al., 2008; Smith, et al., 2008) en un 30\% de los sitios. Esto habla del incremento de esta preocupación en estos espacios singulares.

El 79\% de los casos citan protocolos para la resolución de incidencias que se presenten en el manejo de estos recursos, y el 59 \% de ellos recurre al aviso verbal al coordinador o profesor. La obligatoriedad de un registro inicial de percances implica situar un documento al efecto en cada equipo donde se describa el problema y el usuario. Esta constancia escrita de la incidencia puede favorecer la toma de decisiones y evitar problemas similares futuros.

Por último, el 67 \% de las normas describen algún tipo de corrección ante su incumplimiento por parte del usuario, en el $37 \%$ de los casos la penalización consiste en la suspensión temporal de acceso al aula, y definitiva en el $30 \%$. Cabe observar que el centro educativo tiene unos objetivos centrados en el progreso de la persona, es por ello que las normas también están orientadas a tal fin.

\section{CONCLUSIONES Y PROPUESTAS}

Los reglamentos reúnen indicaciones para las relaciones entre las personas, y las personas con los recursos. Son normas que deben estar de acuerdo con el plan de convivencia, e íntimamente ligadas con las competencias a lograr en el alumnado. El usuario debe ver el aula de informática como continuación del resto de los espacios educativos. En coherencia, las normas razonadas y difundidas deben de marcar el camino para logar objetivos educativos apoyados en los recursos informáticos.

Entre los hallazgos del estudio observamos que las aulas informáticas aún se conciben en su mayoría como lugares cerrados, con mobiliario fijo, estudiantes identificables, dedicados a un modelo didáctico de tipo tradicional. La obligación de conocer y cumplir un conjunto de 
normas parece centrarse más en el cuidado de los equipos y el mobiliario, así como al control de la conducta de los alumnos, y en menor medida en la finalidad de constituir un ambiente propicio para promover el aprendizaje dinámico y colaborativo.

Las normas de informática son cambiantes y se modifican con el devenir tecnológico y las nuevas costumbres de los usuarios. De ahí que este documento pueda ser utilizado para el análisis de las modificaciones subsecuentes en el tiempo, con una visión positiva, proactiva, no punitiva y disuasoria de conductas negativas, con énfasis en el cumplimiento de las metas educativas.

\section{REFERENCIAS}

ACIKALIN, M. (2010). Exemplary social studies teachers use of computer-supported instruction in the classroom. Turkish On line Journal of Educational Technology, 9(4); 66-82.

ANGUITA, M. Y ORDAX, E. (2000). Las alumnas ante los ordenadores; estrategias y formas de trabajo en el aula. Comunicar, 7(14); 218-224.

BENNETT, S., et al. (2008).The 'digital natives' debate; A critical review of the evidence. British Journal of Educational Technology, 39(5); 775-786.

BISCOMB, K., DEVONPORT, T. Y LANE, A. (2008). Evaluating the use of computer-aided assessment in higher education. Journal of Hospitality Leisure Sport y Tourism Education, 7(1); 82-88.

BOWMAN, L., et al. (2010). Can students really multitask? An experimental study of instant messaging while reading. Computers y Education, 54(4); 927-931.

BOZIONELOS, N. (2004). Socio-economic background and computer use; the role of computer anxiety and computer experience in their relationship. International Journal of Human-Computer Studies, 61(5); 725-746.

CARLSON, S. (2005). The next generation goes to college. The Chronicle of Higher Education, 52(7); A34.

COX, S., et al. (2005). Workplace surveillance and employee privacy; Implementing an effective computer use policy. Communications of the IIMA, 5(2); 57-65.

ELLIOTT, M. Y SCACCHI, W. (2005). Free software development; Cooperation and conflict in a virtual organizational culture. Disponible en http://www.ics.uci.edu/ wscacchi/Papers/New/Elliott-Scacchi-BookChapter.pdf (02-04-11).

FARAH, G. V. (2005). La resolución de problemas en matemática y el uso de las TIC: resultados de un estudio en colegios de Chile. Edutec, Revista Electrónica de Tecnología Educativa, 19.

FLOWERS, B. Y RAKES, G. (2000). Analyses of acceptable use policies regarding the Internet in selected K-12 schools. Journal of Research on Computing in Education, 32(3); 351-365. 
GARLAND, K. Y NOYES, J. (2004). The effects of mandatory and optional use on students' ratings of a computer-based learning package. British Journal of Educational Technology, 35(3); 263-273.

GIBERT, G. (2006). Diseño de un aula informática. Tesis doctoral. Universitat Politècnica de Catalunya. Disponible en http://upcommons.upc.edu/pfc/bitstream/2099.1/2991/1/546841.pdf (10-03-11).

GONZALEZ-VIDEGARAY, M., HERNANDEZ-ZAMORA, G. Y DEL-RIO-MARTINEZ, J. (2009). Learning objects in theory and practice: A vision from Mexican University teachers. Computers y Education, 53(4), 1330-1338.

GUTIÉRREZ, A., et al. (2010). Tribus digitales en las aulas universitarias, Comunicar, 17 (34); 173-181.

INAN, F., LOWTHER, D. L, ROSS, S. M. Y STRAHL, D. (2010). Pattern of classroom activities during students' use of computers; Relations between instructional strategies and computer applications. Teaching and Teacher Education, 26(3); 540-546.

ISCIOGLU, E. (2011). Perceived computer self-efficacy of secondary education teachers. New Educational Review, 23 (1); 189-198.

JUDD, T. Y KENNEDY, G. (2011). Measurement and evidence of computer-based task switching and multitasking by 'Net Generation' students. Computers y Education, 56(3); 625631.

JUNCO, R. Y COTTON, S. (2011). Perceived academic effects of instant messaging use. Computers y Education, 56(2); 370-378.

KERR, O. (2011). Ex ante regulation of computer search and seizure. Virginia Law Review, 96(6); 1241-1293.

$\mathrm{KOH}$, J.L. Y FRICK, T. (2009). Instructor and student classroom interactions during technology skills instruction for facilitating preservice teachers' computer self-efficacy. Journal of Educational Computing Research, 40 (2); 211-228.

KOWALSKI, R., et al. (2008). Cyber bullying; Bullying in the digital age, Blackwell Publishing.

$\mathrm{LI}, \mathrm{Q}$. (2007). New bottle but old wine; A research of cyberbullying in schools. Computers in Human Behavior, 23(4); 1777-1791.

LOCATIS, C., et al. (2008). A virtual computer lab for distance biomedical technology education. BMC Medical Education, 8(1); 12.

LÓPEZ, M. V., DAPOZO, G. N., GREINER, C. L. Y ESPÍNDOLA, M. C. (2012). Entorno virtual como soporte a la formación de informáticos en una universidad del Nordeste Argentino. Edutec, Revista Electrónica de Tecnología Educativa, 39.

LOWERISON, G., et al. (2006). Student perceived effectiveness of computer technology use in post-secondary classrooms. Computers y Education, 47(4); 465-489. 
LUND UNIVERSITY LIBRARIES (2009). Directory of Open Access Journals, http;//www.doaj.org/ (04-11-2010).

MARTIN, A., PICOS, A. Y EGIDO, L. (2010). School teacher training and ICT integration in education; anatomy of a mismatch. Revista de Educación, 353, 267-293.

OYEWOLE, S., HAIGHT, J. M. Y FREIVALDS, A. (2010). The ergonomic design of classroom furniture/computer work station for first graders in the elementary school. International Journal of Industrial Ergonomics, 40(4); 437-447.

PEDROSA, M., et al. (2001). La computadora y las actividades del aula; Algunas perspectivas en la educación general básica de la provincia de Buenos Aires. REDIE; Revista Electrónica de Investigación Educativa, 3(2).

PRENSKY, M. (2001). Digital natives, digital immigrants Part 1. On the Horizon, 9(5); 1-6.

ROLANDO, M. Y SALVADOR, CH. (2009). Knowledge System for Application of Computer Security Rules. Conference Information; 2nd International Workshop on Computational Intelligence for Security in Information Systems. Book Series; Advances in Intelligent and Soft Computing, 63; 9-17.

SCHRAW, G. (2010). Measuring self-regulation in computer-based learning environments. Educational Psychologist, 45(4); 258-266.

SELWYN, N. (2007). The use of computer technology in university teaching and learning; a critical perspective. Journal of Computer Assisted Learning, 23 (2); 83-94.

SELWYN, N. (2009). The digital native-myth and reality. Aslib Proceedings; New Information Perspectives, Emerald Group Publishing.

SHELL, D. Y HUSMAN, J. (2008). Control, motivation, affect, and strategic self-regulation in the college classroom; A multidimensional phenomenon. Journal of Educational Psychology, 100(2); 443-459.

SMITH, P. K., et al. (2008). Cyberbullying; Its nature and impact in secondary school pupils. Journal of Child Psychology and Psychiatry, 49(4); 376-385.

SUREDA, J., et al. (2010). Menores y acceso a Internet en el hogar; las normas familiares. Comunicar, 17(34); 135-143.

TONDEUR, J., VAN BRAAK, J. Y VALCKE, M. (2007). Towards a typology of computer use in primary education. Journal of Computer Assisted Learning, 23(3); 197-206.

WILLINGHAM, D. (2010). Have technology and multitasking rewired how students learn? American Educator, 23.

WITTEN, I., et al. (2009). How to Build a Digital Library, Morgan Kaufmann Elsevier Science: San Francisco.

YOUNG, J. (2008). A Computer lab that students use but never see. The Chronicle of Higher Education, 54(38); 1. 
Para citar este artículo:

FOMBONA, J.; GONZÁLEZ, M. del C.; \& GOULGO, M. de F. (2012). Estudio de la normatividad de los espacios informáticos académicos en Iberoamérica. EDUTEC, Revista Electrónica de Tecnología Educativa, 42. Recuperado el dd/mm/aa de http://edutec.rediris.es/Revelec2/Revelec42/estudio normatividad espacios informaticos academicos iberoamerica.html 